Viszeralmedizin 27 | 5 | 11

Fortgeschrittene pankreatische Neuroendokrine Tumoren: mTOR-Inhibitor Afinitor $^{\circledR}$ (Everolimus) erhält EU-Zulassung

Die Diagnose pankreatische Neuroendokrine Tumoren (NET) erfolgt aufgrund der heterogenen Symptomatik oft erst im fortgeschrittenen Stadium. Für diese Situation waren die Therapieoptionen bislang begrenzt. Nun steht europaweit der oral verfügbare MTOR-Inhibitor Afinitor $^{\circledR}$ (Everolimus) zur Behandlung erwachsener Patienten mit inoperablen oder metastasierten, gut oder mäßig differenzierten NET pankreatischen Ursprungs mit progressiver Erkrankung zur Verfügung. Basis für die Zulassung sind die Ergebnisse der plazebokontrollierten Phase-III-Studie RADIANT*'-3: In dieser verlängerte Everolimus signifikant das progressionsfreie Überleben (PFS) bei fortgeschrittenen pankreatischen NET auf mehr als das Doppelte $(p<0,0001)$ [1]. Dabei besitzt der mTOR-Inhibitor ein gut handhabbares Sicherheitsprofil.

Zum Zeitpunkt der Diagnosestellung befinden sich die meisten Patienten mit pankreatischen NET bereits in einem fortgeschrittenen Stadium der Erkrankung, sodass eine operative Entfernung des Tumors nicht mehr möglich ist. In dieser Phase beträgt die mediane Überlebensdauer 2 Jahre [2]. Deshalb ist ein wichtiges Therapieziel, die Krankheitsprogression zu verzögern. Eine wirksame zielgerichtete medikamentöse Behandlung mit einem gut beherrschbaren $\mathrm{Si}$ cherheitsprofil hat daher eine große Relevanz.

Everolimus ist signifikant wirksam bei progredienten fortgeschrittenen pankreatischen NET RADIANT-3 ist die größte, jemals bei pankreatischen NET durchgeführte Studie und Teil des RADIANT-Studienprogramms, welches Wirksamkeit und Sicherheit von Everolimus bei NET evaluiert.
An der prospektiven, randomisierten, doppelblinden, plazebokontrollierten, multizentrischen, zulassungsrelevanten Phase-III-Studie nahmen 410 Patienten mit histologisch gesicherten fortgeschrittenen pankreatischen NET und einem Progress innerhalb der vorangegangenen 12 Monate teil. Die Studienteilnehmer wurden randomisiert im Verhältnis 1:1 und erhielten entweder täglich oral $10 \mathrm{mg}$ Everolimus oder Plazebo, jeweils in Kombination mit Best Supportive Care. Dieses Studiendesign ermöglichte somit eine Therapie mit SomatostatinAnaloga zur Symptomkontrolle, wovon 49 bzw. 50\% der Patienten im Everolimus- bzw. PlazeboArm profitierten [1].

Primärer Endpunkt ist das PFS. Als sekundäre Endpunkte wurden u.a. die objektive Ansprechrate, die Ansprechdauer, das Gesamtüberleben und die Sicherheit ermittelt. Bei einem Fortschreiten der Erkrankung unter Plazebo konnten die Patienten Everolimus erhalten, dies war für 148 von 203 Patienten (73\%) unter Plazebo bei Progress der Fall [1]. In der RADIANT-3-Studie verlängerte Everolimus im Vergleich zu Plazebo signifikant das PFS um das 2,4-Fache - jeweils in Kombination mit Best Supportive Care (11,0 vs. 4,6 Monate; $\mathrm{p}<0,0001)$. Darüber hinaus senkte dieser mTOR-Inhibitor das Risiko der Tumorprogression signifikant um $65 \%(\mathrm{HR}=0,35 ; 95 \%$-KI $0,27-0,45 ; \mathrm{p}<0,0001)$ [1].

Zudem profitierten deutlich mehr Patienten langfristig von der Therapie mit Everolimus im Vergleich zu Plazebo (jeweils in Kombination mit Best Supportive Care): Nach 18 Monaten waren 34,2\% der mit Everolimus behandelten Patienten progressionsfrei (95\%-KI 26-43) gegenüber 9\% unter Plazebo (95\%-KI 4-16) [1].

Ein klinischer Benefit wurde bei $78 \%$ der mit dem mTOR-Inhibitor behandelten Patienten festgestellt: So erzielten $73 \%$ eine Stabilisierung der Erkrankung und 5\% eine partielle Remission [1].
Bei $42 \%$ der Patienten wurde Everolimus als Erstlinientherapie eingesetzt: Auch in dieser Situation verlängerte Everolimus signifikant das PFS auf mehr als das Doppelte (11,4 vs. 5,4 Monate; $p<0,001$ ) [3].

Die beobachteten Nebenwirkungen entsprechen dem bekannten Sicherheitsprofil dieses mTORInhibitors. So wurden in der RADIANT-3Studie folgende Nebenwirkungen mit Grad 3/4 im Verum-Arm am häufigsten beobachtet (Inzidenz $\geq 5 \%$ ): Stomatitis (7\%), Anämie (6\%) und Hyperglykämie (5\%). Diese bedürfen zwar besonderer Aufmerksamkeit, sind jedoch gut beherrschbar. Insgesamt brachen nur 13\% die Therapie mit Everolimus aufgrund von Nebenwirkungen ab [1].

\section{Fazit}

Bislang waren die Therapieoptionen für Patienten mit progredienten fortgeschrittenen pankreatischen NET begrenzt. Mit Everolimus steht für diese Indikation nun eine zielgerichtete medikamentöse Behandlung mit signifikanter PFS-Verlängerung zur Verfügung. Dabei besitzt der mTOR-Inhibitor ein bekanntes, gut beherrschbares Sicherheitsprofil und zeichnet sich durch eine einfache orale Anwendung aus.

RADIANT: RAD001 In Advanced Neuroendocrine Tumors.

\section{Literatur}

1 Yao et al.: N Eng J Med 2011;364:514-523.

2 Yao et al.: J Clin Oncol 2008;26:3063-3072.

3 Pommier et al.: J Clin Oncol 2011;29 (suppl; abstr 4103).

Weitere Informationen bei

Novartis Pharma GmbH

Dr. Irene Roth

Roonstraße 25, 90429 Nürnberg

Tel. +49 911 27312-643, Fax -841

irene.roth@novartis.com

www.novartis.de

\title{
Ticker+++ Ticker+++ Ticker+++ Ticker+++ Ticker+++ Ticker+++ Ticker+++
}

B. Braun Melsungen AG - OPM. Askina Foam von B. Braun präsentiert sich in neuer Qualität. Die Schaumstoffwundauflage Askina Foam zum Einsatz bei stark exsudierenden Wunden verfügt jetzt über einen abgeflachten versiegelten Rand. Durch die Abflachung des Randes werden Scherkräfte minimiert und die Gefahr von Druckstellen wird reduziert. Des Weiteren bietet der abgeflachte Rand einen verbesserten Auslaufschutz. Mehr dazu unter www.wundheilung. bbraun.de.

B. Braun Melsungen AG - OPM

Tel. +49 5661 71-6263

info@bbraun.com
Dr. Loges + Co. GmbH. Morbus Basedow: Neue Studie bestätigt den positiven Einfluss von Selen bei endokriner Orbitopathie. In der aktuell publizierten placebokontrollierten Doppelblindstudie wurde die Effizienz der Selen- mit einer Pentoxifyllin-Therapie verglichen. In der Studie wurde Selen in Form von Natriumselenit eingesetzt. Aus dieser Verbindung (z.B. in selen-loges ${ }^{\circledR}$ ) ist das Spurenelement sofort für den spezifischen Einbau in Selenoenzyme verfügbar. ifemedi

Dr. Jörg Hüve

Tel. +49 4419350590

presse@medizin-ernaehrung.de
Dr. Falk Pharma GmbH. Für Patienten mit akuter Colitis ulcerosa wird die Behandlung weiter vereinfacht: Denn Salofalk ${ }^{\circledR}$ Granu-Stix ${ }^{\circledR}$ von Dr. Falk Pharma ist seit kurzem auch in der $3 g$-Dosierung verfügbar. Dies ist die höchste verfügbare orale Einzeldosis von Mesalazin Das Granulat im 3g-Beutel erlaubt eine einfache einmal tägliche Anwendung - auch in der Akuttherapie.

eickhoff kommunikation

Dr. Maria Ruppert

Tel. +49 221 995951-30

ruppert@medizinmarketingservice.de 


\section{News}

Viszeralmedizin 27 | 5 | 11

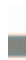

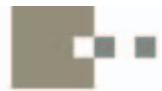

\section{Deutsche Akademie für Mikrotherapie DAFMT}

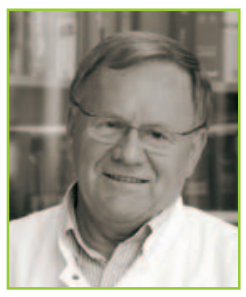

Prof. Dr. Hans Lippert

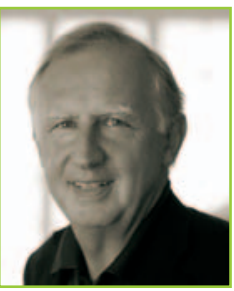

Prof. Dr. Peter Malfertheiner

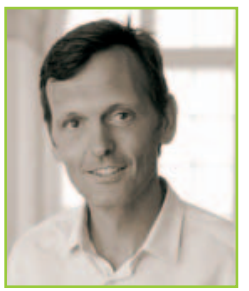

Prof. Dr. Jens Ricke
Mikrotherapien erlangen in der Medizin immer größere Bedeutung: Ob ein Gefäßersatz, eine innere Blutung oder eine Tumorbehandlung - sehr viele Krankheitsbilder lassen sich heute mit minimalen Eingriffen behandeln. Dies erfordert jedoch eine präzise und eingespielte Zusammenarbeit zwischen verschiedenen Fachärzten. Die Deutsche Akademie für Mikrotherapie (DAfMT) hat sich zur Aufgabe gemacht, genau diese interdisziplinären Strategien und Methoden $z u$ vermitteln. Sie versteht sich als Lehreinrichtung für mikrotherapeutische Verfahren, aber auch als Plattform für den internationalen Dialog zwischen Ärzten. Für alle, die an den praktischen Fertigkeiten minimalinvasiver Verfahren interessiert sind, öffnete am 14. Oktober 2011 die Deutsche Akademie für Mikrotherapie ihre Pforten und bereichert so den Standort Magdeburg mit einer weiteren wissenschaftlichen Einrichtung.

«Wir freuen uns sehr, interessierten Ärzten eine moderne Weiterbildungsplattform für minimalinvasive Verfahren bieten zu können. Zur Entstehung der Deutschen Akademie für Mikrotherapie trugen vor allem unsere Bemühungen bei, endlich eine interdisziplinäre Gruppe aufzubauen, die der breiten Ärzteschaft innovative bildgeführte Therapien zugänglich macht», beschreibt Prof. Dr. Jens Ricke, Präsident der DAfMT und Direktor der Klinik für Radiologie und Nuklearmedizin am Universitätsklinikum Magdeburg, die Gründung der Akademie. Gemeinsam mit seinen Kollegen Prof. Dr. Hans Lippert, Stellvertretender Präsident der DAfMT und Direktor der Klinik für Allgemein-, Viszeral- und Transplantationschirurgie, und Prof. Dr. Peter Malfertheiner, Beisitzer des DAfMTPräsidiums und Direktor der Klinik für
Gastroenterologie, Hepatologie und Infektiologie, rief er die DAfMT im Sommer dieses Jahres ins Leben. Die drei Gründer vereinen bereits die geballte Kompetenz verschiedener medizinischer Disziplinen, die durch ein deutschlandweites Netzwerk namhafter Professoren anderer Fachbereiche ergänzt wird. Die Deutsche Akademie für Mikrotherapie kooperiert darüber hinaus mit dem Universitätsklinikum der Otto-von-Guericke-Universität Magdeburg.

Kernelement der Ausbildung an der Akademie sind Kurse, die bewusst über die Fächergrenzen hinweg gehen und das für Mikrotherapien notwendige Wissen kombiniert vermitteln. «Momentan konzentrieren wir uns stark auf die Bereiche interventionelle Radiologie, Endoskopie und minimalinvasive Chirurgie», kommentiert Prof. Lippert das Programm der Akademie. Das Kursspektrum wird in naher Zukunft erweitert. Darüber hinaus betreibt die Akademie präklinische Forschung und Entwicklung im Bereich der Mikrotherapien. Den Stapellauf hat die Deutsche Akademie für Mikrotherapie bereits erfolgreich absolviert: Im Juni fand ein erster Kurs zur Durchführung der Selektiven Internen Radiotherapie (SIRT) statt, die bei Krebs in der Leber zum Einsatz kommt. «Dabei haben wir das Prozedere der SIRT in einer gemischten Gruppe aus Ärzten verschiedener europäischer Nationen trainiert. Diese kamen zum Beispiel aus Belgrad, Rijeka, Ljubljana und Danzig», betont Prof. Malfertheiner die internationale Ausrichtung der Akademie.

Die Gründung der DAfMT ist auch für die Region Magdeburg ein großer Gewinn. «Die Deutsche Akademie für Mikrotherapie steigert das Ansehen unserer Landeshauptstadt als Wissenschaftsstandort», so Dr. Reiner Haseloff, Ministerpräsident von Sachsen-Anhalt. Dr. Haseloff weihte am 14. Oktober 2011 gemeinsam mit über 100 Ärzten, Wissenschaftlern und Unternehmern auf der Burg Wanzleben die Akademie im Rahmen eines Eröffnungssymposiums feierlich ein.

Weitere Informationen bei

Deutsche Akademie für Mikrotherapie c/o ipse Communication

Katrin Lewandowski

Albrechtstraße 14 B

10117 Berlin

Tel. +49 30 288846-14

k.lewandowski@ipse.de 